\author{
LaURa Melosi
}

\title{
VIEUSSEUX E IL GIORNALISMO LETTERARIO E CIVILE
}

\section{ESTRATTO}

$\mathrm{da}$

\author{
GABINETTO SCIENTIFICO \\ LETTERARIO G.P. VIEUSSEUX
}

PENSARE L'ITALIA GUARDANDO ALL'EUROPA

Atti del Convegno di studi

Firenze, 27-29 giugno 2011

A CURA DI

MAURIZIO BossI

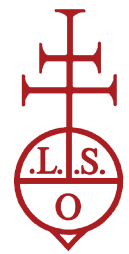

Leo S. Olschki Editore

Firenze 


\section{GIOVAN PIETRO VIEUSSEUX \\ Pensare l'Italia guardando all'Europa}

Atti del Convegno di studi

Firenze, 27-29 giugno 2011

A cura di

Maurizio Bossi

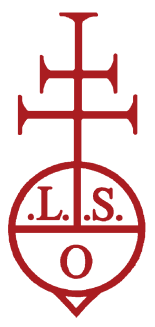

Leo S. Olschki

2013 



\section{GABINETTO G.P. VIEUSSEUX}




\section{GIOVAN PIETRO VIEUSSEUX \\ Pensare l'Italia guardando all'Europa}

Atti del Convegno di studi

Firenze, 27-29 giugno 2011

A cura di

MAurizio Bossi

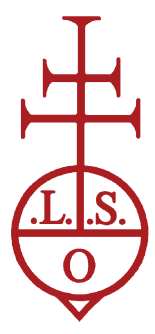

Leo S. Olschki

2013 
Tutti $i$ diritti riservati

Casa Editrice Leo S. OlschKi

Viuzzo del Pozzetto, 8

50126 Firenze

www.olschki.it

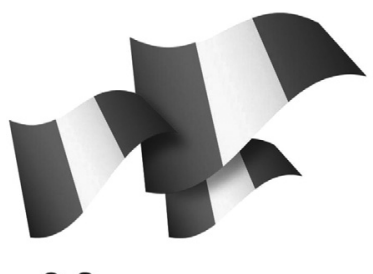

I 86 I $>2$ OII $>>$

I50 anniversario Unità d'Italia

Il volume è pubblicato con il determinante contributo di ENTE

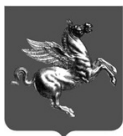

REGIONE TOSCANA

Consiglio Regionale

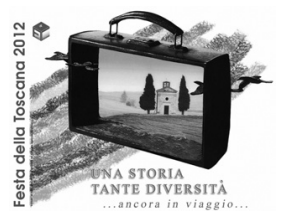

ISBN 9788822262646 


\section{INDICE}

Gloria Manghetti, Premessa. . . . . . . . . . Pag. VII

MAURIzIO BossI, Un'Europa in viaggio. Gli orizzonti di Vieusseux » XI

Luigi Mascilli Migliorini, Orizzonti politico-istituzionali europei per la Toscana della Restaurazione . . . . . . . . . . . . .

Lucia Tonini, Nuove frontiere dell'Europa moderna nella Russia postnapoleonica. I viaggi di Vieusseux, Serristori, Pucci . . . .

Alessandro Volpi, Mercanti e mercato. Dalle merci alla cultura nell'esperienza dei Vieusseux.................. » 25

Francesca Sofia, Sismondi e Vieusseux: le stagioni di un'amicizia 》 41

Marco MANFredi, Religiosità civile nell'Europa di Vieusseux . . » $\quad 59$

Alessandro Breccia, Orientamenti pedagogici europei nell'esperienza del Gabinetto Vieusseux . . . . . . . . . . . . . » 81

FLoriana COlAO, I «giovani legisti» dell' «Antologia». I modelli giuspolitici europei, la Toscana, l'Italia . . . . . . . . . . . » 97

Maria Pia Casalena, Vieusseux e la costruzione della scienza nazionale .....................

Claudio GREPPI, Percorsi di ricerca: fra Humboldt e Darwin. . .

LEONARDO ROMBAI, Le indagini statistiche e la conoscenza del territorio........................

Rossano Pazzagli, Toscana, Europa, Italia: la circolazione delle

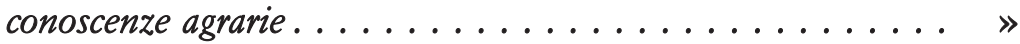

Michele Lungonelli, Le fabbriche Cini. Un modello europeo per l'impresa toscana . . . . . . . . . . . . . . . . . . . .

ANDREA GIUNTINI, Il progresso sui binari. Le prime linee ferroviarie in Europa e in Toscana . . . . . . . . . . . . . . . . . . . 
Laura Melosi, Vieusseux e il giornalismo letterario e civile . . P Pag. 207

Roberto Bigazzi, Storia e romanzo: un problema per l'«Antologia $\ldots \ldots \ldots \ldots \ldots \ldots \ldots \ldots \ldots \ldots \ldots$. 217

CARLO SISI, Correnti artistiche europee e arte nazionale italiana nell' $\ll$ Antologia». . . . . . . . . . . . . . . . . . . .

Elena Marconi, Modelli e suggestioni dell'Europa nella pittura storica toscana ..................... »

\section{PERCORSI DI RICERCA}

\section{INDICE INFORMATIZZATO DEL COPIALETTERE} DI GIOVAN PIETRO VIEUSSEUX

NADA FANTONI, Libri e periodici dall'Europa nei consigli di Vieusseux ai gabinetti di lettura . . . . . . . . . . . . . .

SARA Mori, Censura e circolazione di libri e periodici. Il caso della Toscana granducale (1820-1840) . . . . . . . . . . . » 273

GianluCa Albergoni, Corrispondenti in Lombardia nel Copialettere Vieusseux. Uno sguardo statistico . . . . . . . . . . » 281

Marco Callegari, Giovan Pietro Vieusseux e il Veneto asburgico: rapporti commerciali ed editoriali ............. » 295

Viviana Mellone, La circolazione di opere europee a Napoli tra richieste locali e proposte di Vieusseux . . . . . . . . . 》 305

Manuel PaCe, Circuiti della memoria napoleonica nell'Italia della restaurazione . . . . . . . . . . . . . . . .

Alice De Rensis, Presenze sismondiane nel Copialettere di Vieusseux .......................... » 325

STEFANIA NerozZI, Le esperienze pedagogiche europee di Niccolò Puccini nei rapporti con il Gabinetto Vieusseux . . . . . . . . »

Indice dei nomi. ................ » 


\section{LaURa Melosi}

\section{VIEUSSEUX E IL GIORNALISMO LETTERARIO E CIVILE}

L'originalità del modello giornalistico dell'«Antologia» nel panorama della stampa periodica della Restaurazione è un dato da tempo acquisito alla riflessione storiografica e letteraria. I precedenti immediati e illustri della «Biblioteca Italiana» e del «Conciliatore» sono solo relativamente accostabili alla formula antologica, dato che i due giornali milanesi non le corrispondono né sul piano delle motivazioni genetiche né tanto meno sul piano dell'impresa commerciale. Nel primo caso, si tratta di un organo creato e finanziato dal neorestaurato governo austriaco, allo scopo di riconvertire il consenso nel Lombardo-Veneto dopo che la massiccia utilizzazione della stampa da parte del regime napoleonico aveva formato un'opinione pubblica necessariamente avversa agli antichi regnanti. Nel secondo caso, siamo di fronte a un tentativo di opposizione letteraria nato elitario e destinato a quel 'popolo' di cui fin dal 1816 Berchet aveva delineato con precisione la fisionomia borghese in uno dei più importanti manifesti del romanticismo italiano. ${ }^{1}$

In controluce all'idea di un giornale letterario a Firenze c'è, come sappiamo, il Progetto che Capponi stende in Inghilterra sul finire del 1819, di un foglio periodico che avrebbe dovuto intitolarsi «Archivio di Letteratura». ${ }^{2}$ Il fatto di aver incontrato a Londra Foscolo, a sua volta estensore poco prima dell'abbandono di Milano nel 1815 del celebre Parere sulla istituzione di un giornale letterario, non va considerato ininfluente, visto che quel giornale, con le debite differenze, avrebbe poi effettivamente visto la luce qualche mese

1 G. BERCHET, Sul «Cacciatore feroce» e sulla «Eleonora» di Goffredo Augusto Bürger. Lettera semiseria di Grisostomo al suo Figliuolo, Milano, dai tipi di Gio. Bernardoni 1816 (ora in I manifesti romantici del 1816 e gli scritti principali del «Conciliatore» sul Romanticismo, a cura di C. Calcaterra, Torino, Utet 1964, pp. 267-331).

2 Progetto di giornale, in A. CARRAResI (a cura di), Lettere di Gino Capponi e di altri a lui, V, Firenze, Le Monnier 1882-1887, pp. 93-112. 
dopo nelle forme della filogovernativa «Biblioteca Italiana». Capponi inoltre aveva in prima istanza rivolto l'invito a collaborare proprio a quei letterati che avevano svolto la funzione di redattori nella fase inaugurale della rivista milanese, Vincenzo Monti, Pietro Giordani e Scipione Breislak. Dunque l'ipotesi che il marchese intendesse «ricostruire intorno a sé, assumendone la direzione, il nucleo originario che nel 1816 a Milano aveva dato vita alla "Biblioteca Italiana" ${ }^{3}$ non è senza fondamento, e lo si capisce incrociando i carteggi dei protagonisti della vicenda.

Parallelamente, nella capitale del Granducato si vanno moltiplicando progetti vecchi e nuovi che includono anche Vieusseux fin dall'indomani del suo arrivo a Firenze: dalla ripresa delle pubblicazioni del «Saggiatore» di Lorenzo Collini, che non avrà una seconda chance, ${ }^{4}$ ai tentativi abbandonati ancora in bozze della «Gazzetta letteraria» e del «Giornale Toscano Scientifico e Letterario». Un quesito è sempre rimasto al centro della discussione, se e in quale misura l'«Antologia» abbia costituito la realizzazione del capponiano disegno dell' «Archivio di Letteratura», come per lungo tempo hanno continuato a sostenere gli studi sugli autori che furono a vario titolo della partita. Da Tabarrini a Barbèra, da Gentile a Spadolini la vulgata della identificazione dei due fogli e della trasposizione delle idee del marchese nella rivista diretta da Vieusseux (l'uno la mente, l'altro il braccio esecutivo) si è venuta imponendo, in certi luoghi anche acriticamente, come accade per esempio nei commenti agli epistolari di Monti e di Foscolo dove la si ritrova attestata nelle note rispettivamente del Bertoldi e dello Scotti. Non collimano invece con questo punto di vista la ricostruzione diversamente orientata di Prunas, che parla semmai di una trasformazione operata da Vieusseux del progetto di Capponi, e neppure lo studio di Linaker, l'unico critico a non aver accomunato i due personaggi nella fondazione della rivista. Accertamenti recenti hanno infine chiarito che Capponi prima rinviò e poi rinunciò alla sua impresa per motivi politici, proprio mentre l'«Antologia» cominciava a uscire in forma di 'magazzino', collegato all'attività del gabinetto di lettura con finalità divulgative e promozionali. Al suo esordio, nel gennaio 1821, la rivista di Vieusseux pubblicava solamente traduzioni ed estratti di giornali e di libri stranieri, riprendendo soprattutto dalla «Revue Encyclopédique» di Marc-Antoine Jullien. Anche il disegno dell'«Archivio di Lette-

3 P. NATTA, Una lettera inedita di Pietro Giordani e la paternità dell' «Antologia», «Bollettino Storico Piacentino», LXXXIX, 2, 1994, p. 265.

${ }^{4}$ Il giornale iniziò le pubblicazioni nella prima metà del 1819, ma nonostante il favore di letterati di fama, tra i quali Monti, ebbe vita breve. 
ratura» di Capponi guardava all'Europa, ma viceversa ricalcava i fogli inglesi stile «Quarterly Review».

Pur partendo da impostazioni e modelli di riferimento internazionali diversi, quello che accade nel corso del 1821 è un singolare processo di sintesi, per cui da un certo momento in avanti la fisionomia bibliografica dell'«Antologia» cambia i suoi connotati grazie alla trasfusione di parte dei materiali approntati da Capponi per la sua mai decollata impresa e grazie anche all'ingresso nella redazione di veri professionisti del giornalismo letterario, primo fra tutti il lombardo Giuseppe Montani. A consuntivo del primo anno di pubblicazioni, Vieusseux può dunque scrivere:

ma perché il pubblico avesse sicurtà che io non gli prometteva al di là di quel che le mie forze mi permettevano di attendere mi restrinsi allora a raccogliere semplici traduzioni di estratti di libri e di giornali in lingue oltramontane, riserbandomi a cambiare il mio primo proponimento tosto che la fiducia del pubblico, e la cooperazione de' letterati mel permettessero. ${ }^{5}$

La rappresentazione fornita da Tabarrini di un Capponi maestro di lettere e di un Vieusseux discepolo alla sua scuola appare una semplificazione un po' ingenerosa: al contrario, Vieusseux mercante che si specializza nel commercio librario, che apre un gabinetto di lettura di respiro europeo, che decide di lanciarsi nell'impresa di una rivista, non solo ebbe idee molto chiare su come si dovesse e potesse fare un giornale, ma anche su quali fossero le esigenze letterarie e civili a cui esso doveva rispondere, e lo dimostra ampiamente lo spoglio del suo Copialettere, facilitato ora dall'Indice informatizzato, e del carteggio conservato alla Biblioteca Nazionale di Firenze, ricchissimi entrambi di indicazioni in tal senso. Certo, fu grazie al sostegno di Capponi e degli altri intellettuali che animarono il circolo di Palazzo Buondelmonti se l'«Antologia» colse nel segno, ma al ruolo direttivo esercitato da Vieusseux vanno rivendicati la funzione orientativa e l'incidenza culturale della rivista. Non solo Vieusseux seppe dare un buon giornale alla Toscana, ma l'«Antologia», finché durò, fu l'unico buon giornale che l'Italia potesse allora vantare, come Giordani scriveva a Leopardi nel 1823 facendosi tramite dell'avvio dei rapporti tra il ginevrino e il conte di Recanati. ${ }^{6}$ Provenendo da colui che era stato magna pars nella prima pp. $3-15$.

5 Dal proemio di Vieusseux alla seconda annata della rivista, «Antologia», XIII, gennaio 1822,

6 «L'oggetto mio principale di scriverti da questa beata Firenze [...] è per parlarti di uno de' più bravi e cari uomini che io abbia conosciuti, stabilito da cinque anni in questa città, alla quale ha già fatto un gran bene, e più ancora ne farà; e non solo a Firenze, ma veramente all'Italia: che non avrebbe un buon Giornale, se il signor Giampietro Vieusseux non le avesse data l'Antologia. [...] e io vorrei che tu mandassi materie al suo giornale, che è già senza paragone il migliore (anzi il solo buono) 
redazione della «Biblioteca Italiana», questo giudizio ha un'attendibilità del tutto speciale, anche perché la considerazione che Giordani ebbe sempre del mestiere di giornalista non è affatto incoraggiante. E qui bisogna capire bene la natura di quel cambio di statuto che la figura settecentesca del poligrafo affronta proprio in quest'epoca, una mutazione genetica all'origine della quale c'è ancora l'esperienza della «Biblioteca Italiana». Lo attesta, per esempio, quanto Acerbi scriveva a Giuseppe Carpani, collaboratore specializzato in articoli di argomento artistico:

La cosa che fa più senso, perché non si è mai fatta in Italia, si è il pagamento d'ogni articolo, pagamento che ha già sparso l'allarme fra tutti i giornali letterari e scientifici, i cui editori stanno piuttosto nell'esigere che nel dar pagamento. Sono curioso di vedere l'effetto, che il nostro farà su di essi. Qualche editore mi ha già fatto parlare per pattuire, offrendomi d'abbandonare la sua impresa per associarsi alla nostra. ${ }^{7}$

Come ha ben spiegato Marino Berengo, ${ }^{8}$ si trattava di una rivoluzione nel modo di concepire la professione giornalistica, dettata anche dalle condizioni di necessità in cui si trovarono a operare molti uomini di cultura esuli o disoccupati all'indomani della caduta del Regno d'Italia. Giordani stesso ne sapeva qualcosa, per essere passato di punto in bianco dal ruolo di funzionario dell'amministrazione napoleonica a quello di libero professionista in cerca di ingaggio, e non per nulla dopo la deludente esperienza di «pasticcere e lavandaia» della «Biblioteca Italiana», si era convinto della necessità di sostenere la ricchezza e la nobiltà come requisiti essenziali del letterato, perché nella sua visione niente affatto anacronistica del problema queste prerogative si ponevano a garanzia della funzione civile dello scrittore e della sua libertà di espressione. Lo aveva detto in confidenza a Leopardi in una lettera del settembre $1817 ;^{9}$ aveva poi formulato

d'Italia; e che si farà ottimo, se i migliori d'Italia si uniranno tutti a lui. La Censura di Firenze è la più benigna in tutta Italia: il Direttore Vieusseux è il solo che intenda che cosa sia, e come debba esser fatto un buon giornale. Tu che hai il più raro ingegno che io mi conosca, e tanto sapere che appena è credibile; potrai farti conoscere così stupendo come sei, in questo giornale, che è il solo che abbia credito». Lettera di Giordani del 5 novembre [1823], in G. LEOPARDI, Epistolario, I, a cura di F. Brioschi e P. Landi, Milano, Bollati Boringhieri 1998, pp. 752-753.

7 A. Galante Garrone, I giornali della Restaurazione, in ID. - F. Della Peruta, La stampa italiana del Risorgimento, Bari, Laterza 1979, p. 24. Sulla collaborazione alla «Biblioteca Italiana» di Carpani, «esemplare da museo di reazionario perfetto» ma non «passatista ottuso», cfr. R. BizzoCCHI, La «Biblioteca Italiana» e la cultura della Restaurazione (1816-1825), Milano, Franco Angeli 1979, pp. 99 sgg.; se ne veda anche la recensione di R. TissonI, La «Biblioteca Italiana» e la cultura della Restaurazione nel Lombardo-Veneto, «Studi storici», XXI, 2, 1980, pp. 421-436.

8 M. BERENGo, Intellettuali e librai nella Milano della Restaurazione, Torino, Einaudi 1980.

9 «Per pietà, per tutte le care cose di questo mondo e dell'altro, ponete, mio carissimo Contino ogni possibile studio a conservarvi la salute. La natura lo ha creato, voi l'avete in grandissima parte 
questo principio nel saggio del 1821 sul Perfetto scrittore italiano e lo difendeva qualche anno dopo dagli attacchi di cui era stato fatto oggetto, richiamando la questione nel manifesto della «Scelta di Prosatori Italiani», la collana di classici che Giordani e Vieusseux avevano progettato insieme nel 1825: «E perché so che il mio scrittore dev'essere odiato da' tristi; ma non voglio che possa essere oppresso; domandavo alla fortuna che non gli negasse nobiltà né ricchezze». ${ }^{10}$

Questa concezione del giornalismo letterario rende ragione della tutto sommato labile collaborazione di Giordani alla rivista di Vieusseux, nonostante il direttore avesse puntato sull'esule piacentino fin dall'arrivo di quest'ultimo a Firenze nel 1824. La prospettiva da cui Giordani guardò a questo impegno non corrispose fin dall'inizio al modello pensato da Vieusseux per i collaboratori della sua rivista, e ciò principalmente per le riserve verso un tipo di produzione letteraria che imponeva tempi di scrittura compressi, a detrimento della qualità dello stile e della profondità della riflessione con cui gli argomenti avrebbero dovuto essere trattati. Su questo punto occorre ricordare che il classicista Giordani, intellettuale impegnato ma di formazione accademica, si trovava in perfetta sintonia con le idee che anche Leopardi ebbe riguardo alla stampa periodica e in particolare alle gazzette, lettura «di un momento» fatta oggetto di ripetuti attacchi satirici nelle sue opere. Tristano che con finta serietà professa: «Credo ed abbraccio la profonda filosofia de' giornali, i quali uccidendo ogni altra letteratura e ogni altro studio, massimamente grave e spiacevole, sono maestri e luce dell'età presente», parla all'unisono con l'autore della Palinodia al marchese Gino Capponi: le gazzette, «... anima e vita / dell'universo, e di savere a questa / ed alle età venture unica fonte!» ( $v v .151-153)$.

Quanto a Giordani, è da un tale disincanto che deriva in gran parte la sua esigenza di mantenersi libero da vincoli contrattuali di qualunque sorta, nonostante ancora nel marzo 1825, al momento di negoziare l'impegno per la

lavorato quel perfetto scrittore italiano che io ho in mente. Per dio, non me lo ammazzate. Dovete sapere che nella mia mente è fisso che il perfetto scrittore d'Italia debba necessariamente esser nobile e ricco. Né crediate che sia adulazione: ché anzi la vostra dolcezza si spaventerebbe se sapesse a qual segno io fierissimamente disprezzo più d'ogni altra canaglia i nobili, quando sono asini e superbi. Ma per molte ragioni, che un giorno dirò a stampa, io vorrei che la maggior parte de' nobili fosse virtuosa e culta; parendomi questa l'unica ragionevole speranza di salute all'Italia. E poi tante cose dee sapere e volere e potere lo scrittore perfetto, che non può volere e potere e sapere se di nobiltà e ricchezza non sarà munito». Lettera di Giordani del 21 settembre [1817], in G. LEOPARDI, Epistolario, I, cit., pp. 140-141.

10 Scritti editi e postumi di Pietro Giordani pubblicati da Antonio Gussalli, IV, Milano, Borroni e Scotti 1857, p. 93. Sul progetto editoriale della «Scelta di Prosatori Italiani» di Giordani e Vieusseux sia consentito rinviare al mio In toga e in camicia. Scritti e carteggi di Pietro Giordani, Lucca, Pacini Fazzi Editore 2002, pp. 51-72. 
«Scelta di Prosatori», Vieusseux fosse tornato a prospettargli l'ipotesi di una collaborazione regolare e retribuita: «E giacché siamo sul particolare dell'interesse - gli scriveva il 15 marzo -, permettetemi di far discorso anche per gli articoli che somministrerete all'avvenire dell' "Antologia" pei quali (approvati che siano dalla censura) vorrei vedervi accettare L. 50. per ogni foglio di stampa». ${ }^{11}$ Agli occhi di Giordani, dopo l'esperienza della «Biblioteca Italiana» che ai redattori garantiva da contratto una «ricognizione» fissa di 1.200 franchi l'anno e la partecipazione all'eventuale «vantaggio» derivato dal sopravanzare degli abbonamenti rispetto alle unità prefissate, ${ }^{12}$ la questione del pagamento degli articoli si pose in termini ideologici più che deontologici. Homme de lettres per vocazione e formazione, lo scrittore era ben deciso, ora che poteva permetterselo, a sottrarsi a un rapporto di natura economica che inevitabilmente avrebbe finito per limitare la sua autonomia di pensiero. A prescindere dunque dalla fiducia riposta in Vieusseux, il rifiuto era la via che sceglieva per garantirsi un'incondizionata libertà di giudizio (censure governative a parte), e difatti così si giustificava con Brighenti in una lettera datata 26 febbraio 1825 :

O come volete imaginarvi che [Vieusseux] abbia da poter comandare a me che non mi lascio comandar da nessuno! Che se scrivendo nell'Antologia, cedo al suo volere; la cosa è diversa. L'Antologia è un giornale già stabilito; il direttore, il padrone è lui. Non essendo io pagato, non ci può essere sospetto di viltà quando mi adatti al suo genio. ${ }^{13}$

Particolarmente eloquente, sul piano opposto, è quanto invece succede in quello stesso 1825 con Tommaseo, che bussa alla porta di Vieusseux per offrirgli «in servigio» la sua «incognita penna». ${ }^{14}$ Vieusseux ci mette due mesi a rispondergli, poi vuol esser certo che il candidato coltivi le scienze morali e

11 Carteggio Giordani-Vieusseux 1825-1847, a cura di L. Melosi, Firenze, Olschki 1997, p. 51.

12 Cfr. R. ScHIPPISI, La collaborazione del Giordani alla «Biblioteca Italiana», in ID., Capitoli giordaniani, Piacenza, Tip.Le.Co. 1992, pp. 16 e sgg. Si veda inoltre G. CECIONI, La partecipazione alla «Biblioteca Italiana», in ID., Lingua e cultura nel pensiero di Pietro Giordani, Roma, Bulzoni 1977, pp. 111-154.

13 Epistolario di Pietro Giordani edito per Antonio Gussalli compilatore della vita che lo precede, V, Milano, Borroni e Scotti 1854, pp. 384-385. Si ricordi che a questa data Giordani aveva già pubblicato nell' «Antologia» due articoli d'arte, Sulla Carità modellata da Lorenzo Bartolini, XV, settembre 1824, pp. 90-93 e Della pittura in porcellana, XVI, dicembre 1824, pp. 124-132, e la lettera al marchese Capponi sulla «Scelta di Prosatori Italiani», evidentemente senza ricevere alcun compenso. Nel Copialettere di Vieusseux, di natura prevalentemente commerciale, non si riscontrano pagamenti all'ordine di Giordani.

14 N. Tommaseo - G.P. Vieusseux, Carteggio inedito (1825-1834), a cura di R. Ciampini e P. Ciureanu, Roma, Edizioni di Storia e Letteratura 1956, p. 15, lettera del 10 settembre 1825. 
politiche, e solo dopo aver ricevuto assicurazioni in tal senso («Gli argomenti morali e politici son quelli ch'io meglio amerei; non negatami qualche breve scorreria nelle regioni del Bello») ${ }^{15}$ gli propone di collaborare a patti chiari:

Riguardo alle condizioni, Ella sa che siamo in Italia, paese miserabile per i giornalisti: gli basti che appena cuopro le mie spese. Trenta franchi, per un foglio di stampa del mio giornale, ecco ciò che posso offrirle. Ogni trimestre si farebbero i conti, e le rimetterei il suo avere con una cambialina per Milano. ${ }^{16}$

Prende così avvio una collaborazione intensa, croce e delizia del direttore dell'«Antologia» che con K.X.Y. dovette usare molta pazienza:

Mio caro Tommaseo, voi vi lasciate abbagliare dalla facilità colla quale scrivete. Vi sarebbe poco male se si trattasse di riempiere le colonne dell'Osservatore veneziano, o di tutt'altra gazzetta quotidiana; ma per un giornale come l'Antologia, conviene provvedere con più lentezza. In pochi giorni avete scritto tre o quattro articoli più $o$ meno lunghi. Il Montani, per parlar di quel primo volume ciceroniano, mi avrebbe chiesto almeno un mese di tempo. Voi mi risponderete che per 30 franchi il foglio non si può stare più settimane sopra 24 pagine; e voi avete ragione sino ad un certo segno. Ma se le mie circostanze mi avessero permesso di farvi migliori condizioni, non vi avrei consigliato di restare in Lombardia: vi avrei incoraggiato a portarvi in Toscana. Del resto, quest'articolo vi sarà portato in conto, come se lo avessi stampato; e voi dal canto vostro mi manderete, sul medesimo primo volume, un altro articolo, più breve e meno dotto, ma più filosofico, e (forse lo giudicherete convenevol cosa) scritto in un modo un poco più rispettoso per la memoria di Marco Tullio, que vous avez traité un peu légèrement. ${ }^{17}$

Le perplessità di Giordani sulla scrittura giornalistica non erano dunque del tutto fuori luogo. Ritorniamo però al punto centrale della questione. Si deve ancora al Parere di Foscolo l'indicazione dell'enorme importanza che avrebbe avuto l'assunzione dell'intero onere finanziario da parte della direzione del giornale, senza associare all'impresa stampatori, editori o librai che avrebbero potuto trasformarla in una mera speculazione mercantile. Nella «Biblioteca Italiana» le cose erano andate in un altro modo, per via della sovrapposizione di Vienna all'iniziativa privata, che aveva creato attraverso sov-

15 Ivi, p. 16, lettera del 6 novembre 1825.

16 Ivi, p. 17, lettera dell'8 dicembre 1825.

17 Ivi, p. 57, lettera del 7 ottobre 1826. Sulla collaborazione di Tommaseo all'esperienza dell'«Antologia», cfr. A. VolPI, Alla ricerca del giornalista ideale: la collaborazione di Niccolò Tommaseo con Giovan Pietro Vieusseux, in R. TURCHI - A. Volpi (a cura di), Niccolò Tommaseo e Firenze, Firenze, Olschki 2000 ( «Gabinetto Scientifico Letterario G.P. Vieusseux - Centro romantico - Studi», 9). 
venzioni dirette e sottoscrizioni forzose le condizioni per una circolazione della rivista ampia e diffusa. Nel caso dell'«Antologia», invece, direttore editore e mercante coincisero nella figura unica di Vieusseux, e ciò contribuì in maniera decisiva al successo dell'impresa. L'altro elemento di fondamentale importanza indicato da Foscolo era la commisurazione al successo delle vendite delle retribuzioni da corrispondere agli estensori, possibilmente i migliori sulla piazza, che in questo modo venivano a configurarsi come una vera e propria categoria professionale. E Vieusseux, che conosceva bene tanto il mercato quanto gli uomini, aveva di sua iniziativa applicato il principio alla lettera, perché sapeva per esperienza che «il dire ad uno scrittore voi riceverete una giusta somma per ogni foglio, somma la quale crescerebbe a seconda dell'interesse della materia, è un dire che non può sodisfare l'amor proprio dello scrittore». Meglio allora proporre: «per cominciare darò $£ 36$ a 40 il foglio di 16 pagine, e se le cose andranno bene per l'intrapresa sarò io il primo interessato a offrire un prezzo maggiore dei vostri lavori». ${ }^{18}$

La linea editoriale, la garanzia economica e il controllo sulle vendite dell'«Antologia» furono costantemente e saldamente nelle mani di Vieusseux. L'intera storia della rivista, se scritta sui documenti d'archivio, lo attesta senza possibilità di fraintendimenti e lo si capisce ancora meglio considerando ciò che accadde subito dopo la soppressione decretata dalla censura granducale. Fra il 1833 e il 1834 Vieusseux collaborò con Giuseppe Ricciardi per la diffusione in Italia del «Progresso delle Scienze delle Lettere e delle Arti», cercando di dirottare gli associati al suo giornale su quello napoletano e sostenendo l'iniziativa del conte di Camaldoli con materiali e suggerimenti pratici, frutto della sua ultradecennale esperienza. Ne danno testimonianza alcune lettere già pubblicate nella raccolta Leopardi nel Carteggio Vieusseux ${ }^{19} \mathrm{e}$ una ventina di missive inedite del Copialettere, piene al solito di cifre e indicazioni di natura commerciale, ma anche di consigli di natura editoriale che nel loro insieme offrono una sintesi particolarmente efficace del modo di lavorare di Vieusseux. Cessata l'«Antologia» alla fine del 1832, nell'ottobre 1833 l'ex direttore spedisce una circolare di presentazione del «Progresso» ai 629 associati che aveva raccolto nei vari stati, invitandoli a sottoscrivere

18 Archivio Storico Gabinetto Vieusseux (ASGV), XIX, 1A.5, p. 224.

19 Leopardi nel Carteggio Vieusseux. Opinioni e giudizi dei contemporanei 1823-1837, a cura di E. Benucci, L. Melosi, D. Pulci, Firenze, Olschki 2001 («Gabinetto Scientifico Letterario G.P. Vieusseux - Centro Romantico - Studi», 7): lettere CCLI, CCLII, CCLV, CCLVI, CCLVII, CCLVIII. Le missive provengono dal Fondo Vieusseux della Biblioteca Nazionale Centrale di Firenze, oggetto negli anni Novanta di un'ampia ricognizione sulle tracce di Leopardi, promossa e diretta dal Centro Romantico. 
l'abbonamento al periodico di Ricciardi. ${ }^{20}$ A dicembre gli abbonati sono 116, nel mese seguente 183 , con la previsione di toccare presto quota $200 .^{21}$ Per intensificare la campagna delle sottoscrizioni, Vieusseux suggerisce a Ricciardi di fornirgli delle copie del «Progresso» da regalare e gli propone di scambiare la rivista con le altre italiane, l'«Indicatore», la «Biblioteca Italiana», gli «Annali di Statistica e Tecnologia», gli «Annali delle Scienze di Padova», il «Giornale delle belle arti» di Venezia, «Il Poligrafo» di Verona, il «Giornale Arcadico» di Roma, il «Giornale dei Letterati» di Pisa. Per meglio sostenere le finalità intellettuali del suo progetto, Ricciardi dovrebbe poi tenersi aggiornato sulle novità europee attraverso i maggiori giornali stranieri, di cui segnalare gli articoli più importanti: «Quarterly Review», «Westminster Review», «Revue germanique», «Revue Britannique», «Revue d'Economie politique», «Revue des deux mondes», «Revue de Paris», «Annales de la Marine», «France Littéraire», «Annales des sciences naturelles», «Annales des voyages», «Revue Enciclopedique». Passano poi alla redazione di Napoli una serie di articoli che Vieusseux aveva in cantiere, per esempio quelli a firma K.X.Y. che nel «Progresso» dovranno essere esplicitamente firmati Niccolò Tommaseo. Peccato però che essi incappino nelle maglie della censura, «cosa disastrosa», commenta Vieusseux, ${ }^{22}$ che a quel punto consiglia a Ricciardi di chiedere ai collaboratori contributi che non corrano il rischio di essere bloccati dalle autorità.

Per mesi Vieusseux e Ricciardi discutono di novità librarie da recensire e di miglioramenti da apportare al giornale nella cadenza, nel formato e nella veste grafica. ${ }^{23}$ Vieusseux condivide le proposte di Ricciardi, ma gli racco-

20 Lettera del 15 ottobre 1833, ASGV, XIX 1A.5, pp. 224 e sgg.: «Qui annesso vi rimetto la minuta della circolare che io vorrei mandare ai miei antichi associati [...] la prudenza per non espormi a dispiacere a chi soppresse l'Antologia [...] D'altronde il titolo del Giornale, il vostro nome e, mi sia lecito il dirlo, il mio in fondo, dicono abbastanza per ispirare ogni fiducia al pubblico italiano. [...] direte che le associazioni per Firenze e per tutta la Toscana si prendono al mio gabinetto [...]. Resta bene inteso poi che quando le circostanze diventassero meno dolorose per me, e quando disposizioni più miti a mio riguardo per parte del Governo toscano mi permettessero di intraprendere io medesimo un altro giornale, saremmo liberi di sciogliere il nostro [illeggibile] contratto. Ma secondo tutte le speranze ciò non accadrà tanto presto; e poi avrei tutt'altro in mente che un giornale mensile, cosicché è probabile che la vostra e la mia intrapresa come due sorelle senza farsi torto [illeggibile]. Ad ogni modo vi consiglio pel vostro proprio interesse di preparare, per premetterlo al fascicolo di Gennaio, un discorso nel quale vi intenderete su cosa si debba intendere per progresso dell'Umanità. Io potrei rimettervi copia di ciò che in questo proposito era stato scritto per l'Antologia quando il vostro savio e intelligente Censore vi lasciasse presentare al pubblico una simile franca sì, ma [illeggibile] religiosissima ed umanissima dichiarazione, sareste sicuri di farvi applaudire dall'Italia tutta».

21 I dati si ricavano dalla lettera di Vieusseux del 26 dicembre 1833, pubblicata in Leopardi nel Carteggio Vieusseux, cit., p. 429 e dalla lettera dell'1 febbraio 1834, ASGV, XIX 1A.5.

22 ASGV, XIX 1A.5, lettera del 12 aprile 1834.

${ }^{23}$ Ivi, lettera del 2 luglio 1834. 
manda di essere cauto e di non promettere ai lettori cose impossibili da mantenere «in un paese di non libera stampa», ${ }^{24}$ come per esempio l'impegno a far conoscere tutte le opere interessanti che verranno pubblicate in Italia e all'estero, considerando gli alti dazi sui libri e quindi la difficoltà di reperirli. Quanto alle velleità autoriali di Ricciardi, a giudizio di Vieusseux il direttore di un giornale non dovrebbe mai scrivere articoli, per mantenere libertà e indipendenza di giudizio sugli scritti degli altri. Lo aveva sperimentato durante la direzione dell' «Antologia»: se letterati anche insigni avevano accettato le sue obiezioni e i suoi rifiuti era stato per il ruolo di arbitro super partes che egli aveva saputo ritagliare a se stesso, guadagnandone assai in autorevolezza.

Eppure, nonostante il forte impegno, alla fine del luglio 1834 Vieusseux si trova a dover chiarire alcuni punti relativi alla sua collaborazione: intanto non può garantire di inviare un certo numero di articoli l'anno perché gli scrittori toscani temono la censura, sono attirati dai giornali di Milano e sono scarsamente retribuiti. Non può nemmeno impegnarsi a tenere Ricciardi al corrente delle novità dell'Italia settentrionale in fatto di scienze e di lettere, visto che non c'era riuscito neanche con l'«Antologia». Infine, non è più in grado di offrire garanzie sul numero degli associati, che cominciano a tirarsi indietro. A conti fatti questa intermediazione è piena di difficoltà economiche e burocratiche che si risolvono per lui in un'esposizione finanziaria eccessivamente pesante, perché fino a quel momento ha rimesso a Napoli più di quanto abbia riscosso e le prospettive non sono affatto confortanti.

Cala dunque il sipario su questo nuovo tentativo imprenditoriale di Vieusseux e siccome a Firenze le difficoltà continuano a essere insormontabili e non ci sono accenni di distensione del clima, non resta che sperare in un rinnovamento delle condizioni politiche, in un futuro non troppo lontanto che consenta a un'impresa giornalistica di impostazione progressista un margine accettabile di libertà intellettuale. L'appuntamento è rinviato a quello che nel decennio a seguire sarà l'«Archivio Storico Italiano».

${ }^{24}$ Ivi, lettera del 31 luglio 1834, anche per le notizie a seguire. 



\section{CDC \\ artilgrafiche}

CITTÀ DI CASTELLO • PG

FINITO DI STAMPARE NEL MESE DI OTTOBRE 2013 

ISBN 9788822262646 

